\title{
Annotations
}

\section{The cystic fibrosis gene}

In a clutch of three papers from a recent issue of Science scientists from Toronto and Michigan describe the isolation, sequence, and major mutation of the gene at the cystic fibrosis locus. ${ }^{1-3}$ These reports represent the culmination of several years painstaking work by many scientists worldwide and herald a new era of multidisciplinary effort aimed at devising a treatment for this life limiting condition.

The gene itself was pinned down by extensive genetic analysis of families with cystic fibrosis from many countries; it is likely that the limits of genetic resolution had almost been reached. With the gene bracketed by two DNA markers Dr Lap-Chee Tsui (Hospital for Sick Children, Toronto), Dr Francis Collins (University of Michigan), and their collaborators utilised the technique of 'chromosome jumping'. This allowed them to move over large distances of a chromosome (of the order of 100000 basepairs of DNA, $100 \mathrm{~kb}$ ) relatively quickly. After every jump the position of each clone with respect to the gene defective in cystic fibrosis $(C F)$ was checked using genetic and physical mapping methods. Clones close to $C F$ were tested for the presence of genes which were expressed in tissues known to be affected by the disease; one gene was isolated which mapped to the area suspected of harbouring $C F$ and which was expressed in lung, liver, sweat gland, colon, and pancreas.

The candidate gene is large, extending over 250 $\mathrm{kb}$ of genomic DNA encoding an mRNA of $6.5 \mathrm{~kb}$ constructed from 24 exons (regions of translated sequence separated by non-coding introns). In order to obtain further evidence that this might be $C F$ the sequence of the gene from affected and heterozygote individuals was compared and a deletion of three base pairs of DNA was found in the sequence from chromosomes carrying $C F$. The deletion was found to alter the structure of a protein involved in membrane transport processes.

The sequencing data yielded much other interesting data. The predicted protein sequence of the gene (dubbed cystic fibrosis transmembrane conductance regulator, CFTR) is similar to that of a known gene family encoding the P-glycoproteins alternatively known as multiple drug resistance (MDR) proteins. These proteins were isolated in cells that had become resistant to high concentrations of cytotoxic drugs where they are expressed in huge amounts secondary to gene amplification. The MDR genes encode proteins that span the cell membrane and pump the toxic agents out of the cell in an ATP dependent fashion. The function of the MDR family in the normal cell is unknown; one possibility is that they excrete toxic organic compounds from the cell, another is that they export proteins from the cell.

The discovery of a transmembrane protein encoded at $C F$ is not a complete surprise; a series of elegant experiments has demonstrated a deficiency in chloride ion permeability in the apical membranes of epithelia in cystic fibrosis. After certain (nonphysiological) stimuli the channel for chloride ions found in membranes from cystic fibrosis patients can be made to function in an essentially normal way, demonstrating that the anion transporting domain of the protein is intact but that the regulation of its activity is defective. ${ }^{4}$ CFTR may be a novel type of channel which transports chloride, and perhaps other substrates, in response to intracellular stimuli such as raised cAMP levels and protein kinase activity. Alternatively, the protein may not itself transport chloride, but regulate the activity of the protein that does. The three base pairs at the deletion found in cystic fibrosis encodes a phenylalanine residue which will be absent from the protein in cells in cystic fibrosis; the mutation leaves the rest of the mRNA in frame for translation so that the remainder of the protein is intact. One current hypothesis is that the deletion of this single amino acid results in a conformational change that renders the protein refractory to the various activators of chloride permeability.

It was already thought likely from previous studies that two or more different mutations, in the same gene, may cause cystic fibrosis. ${ }^{56}$ The sequencing of CFTR from various people with cystic fibrosis supports this view. A total of $68 \%$ of chromosomes carrying $C F$ had the 3 bp deletion in CFTR; work is currently underway to determine the nature of the other mutations. The $3 \mathrm{bp}$ deletion was never found on the normal chromosomes of obligate heterozygotes. It is of interest that patients with cystic fibrosis who have pancreatic sufficiency tend to have one chromosome with the 3 bp deletion and one chromosome with another, as yet unknown, 
mutation: that is, they are compound heterozygotes. Patients with pancreatic insufficiency are very often homozygous for the $3 \mathrm{bp}$ deletion, suggesting that either this mutation alters protein function more severely than the others or that the Phe residue is particularly important for functional regulation in the pancreas.

At the clinical level the immediate benefits of this advance are, once all the mutations are characterised, antenatal diagnosis with a theoretical accuracy of $100 \%$ and carrier detection for random members of the population wishing to marry those known to be heterozygous for cystic fibrosis. In the same way it will be possible to confirm a diagnosis of cystic fibrosis in doubtful cases. Diagnostic difficulty, however, is more likely to occur in milder forms of the disease for which the mutation has not yet been determined. With the availability of the polymerase chain reaction it is now possible to detect the major mutation in cystic fibrosis using minute quantities of blood. Should this procedure become automated a method of neonatal screening/heterozygote detection would be feasible using the blood on a Guthrie card With the necessary political and financial will it wil be possible to introduce population screening for the cystic fibrosis mutation, which may make a large impact in reducing the incidence of the disease in the future.

In the longer term the aims will be to devise a treatment for existing and future patients with cystic fibrosis. Pharmacological agents will be sought that modify the activity of CFTR and restore apical membrane chloride permeability, although this is likely to be a difficult task. Several groups will investigate the possibility of introducing a normal CFTR gene into affected cells using viruses tropic for the cell type in question. Mouse models of cystic fibrosis will need to be created so that any new therapeutic approach can be tested in animals. This will be attempted by mutating the mouse homologue of CFTR and breeding mice homozygous for the cystic fibrosis mutation.

As is usual in these situations this latest break through raises more questions than it answers, and certainly there is no prospect of an early 'cure' for cystic fibrosis. There are almost immediate clinical benefits in the areas of diagnosis and heterozygote detection, however, and at least we now know what questions to ask in the search for a complete understanding of the pathophysiology and eventual effective treatment of cystic fibrosis.

\section{References}

1 Rommens JM, Iannuzzi MC, Kerem B-S, et al. Identification of the cystic fibrosis gene: chromosome walking and jumping. Science 1989;245:1059-65.

2 Riordan JR, Rommens JM, Kerem B-S, et al. Identification of the cystic fibrosis gene: cloning and characterisation of complementary DNA. Science 1989;245:1066-73.

3 Kerem B-S, Rommens JM, Buchanan JA, et al. Identification of the cystic fibrosis gene: genetic analysis. Science 1989;245: 1073-9.

4 Schoumacher RA, Shoemaker RL, Halm DR, Tallant EA, Wallace RW, Frizzell RA. Phosphorylation fails to activate chloride channels from cystic fibrosis airway cells. Nature 1987;330:752-4.

${ }^{5}$ Estivill X, Scambler PJ, Wainwright BJ, et al. Patterns of polymorphism and linkage disequilibrium for cystic fibrosis. Genomics 1987;1:257-63.

${ }^{6}$ Kerem B-S, Buchanan JA, Durie P, et al. DNA marker haplotype association with pancreatic sufficiency in cystic fibrosis. Am J Hum Genet 1989;44:827-34.

\section{P J SCAMBLER Cystic Fibrosis Research Group, Department of Biochemistry and Molecular Genetics, St Mary's Hospital Medical School, Norfolk Place, London W2 1PG}

